Filippos T. Filippidis $\odot^{1}$, Vasiliki Gerovasili ${ }^{2}$, William D-C. Man $^{3}$ and Jennifer K. Quint ${ }^{4}$

${ }^{1}$ Dept of Primary Care and Public Health, School of Public Health, Imperial College London, London, UK. ${ }^{2}$ Dept of Respiratory Medicine, Harefield Hospital, Royal Brompton \& Harefield NHS Foundation Trust, London, UK. ${ }^{3}$ NIHR Respiratory Biomedical Research Unit, Royal Brompton \& Harefield NHS Foundation Trust and Imperial College London, London, UK. ${ }^{4}$ Dept of Respiratory Epidemiology, Occupational Medicine and Public Health, National Heart and Lung Institute, Imperial College London, London, UK.

Correspondence: Filippos T. Filippidis, Dept of Primary Care and Public Health, Imperial College London, The Reynolds Building, St Dunstan’s Road, London W6 8RP, UK. E-mail: f.filippidis@imperial.ac.uk

Received: April 182016 | Accepted after revision: Aug 02 2016 | First published online: Oct 062016

Conflict of interest: None declared.

\title{
References
}

$1 \quad$ Hellenic Statistical Authority. Statistics 2016. www.statistics.gr Date last accessed: January 28, 2016.

2 Simou E, Koutsogeorgou E. Effects of the economic crisis on health and healthcare in Greece in the literature from 2009 to 2013: a systematic review. Health Policy 2014; 115: 111-119.

3 Kentikelenis A, Karanikolos M, Reeves A, et al. Greece's health crisis: from austerity to denialism. Lancet 2014 383: 748-753.

4 Tarantilis F, Athanasakis K, Zavras D, et al. Estimates of price and income elasticity in Greece. Greek debt crisis transforming cigarettes into a luxury good: an econometric approach. BMJ Open 2015; 5: e004748.

5 Filippidis FT, Schoretsaniti S, Dimitrakaki C, et al. Trends in cardiovascular risk factors in Greece before and during the financial crisis: the impact of social disparities. Eur J Public Health 2014; 24: 974-979.

6 Euromonitor International. Passport Database 2016. http://go.euromonitor.com/passport.html.

7 European Commission. Special Eurobarometer 429. Attitudes of Europeans towards tobacco and electronic cigarettes. http://ec.europa.eu/public_opinion/archives/ebs/ebs_429_en.pdf.

8 Paraskevopoulou D, Liakakou E, Gerasopoulos E, et al. Sources of atmospheric aerosol from long-term measurements (5 years) of chemical composition in Athens, Greece. Sci Total Environ 2015; 527-528: 165-178

9 U.S. Department of Health and Human Services. The Health Consequences of Smoking-50 Years of Progress. A Report of the Surgeon General, 2014. http://www.surgeongeneral.gov/library/reports/50-years-of-progress/.

10 Hoek G, Krishnan RM, Beelen R, et al. Long-term air pollution exposure and cardio-respiratory mortality: a review. Environ Health 2013; 12: 43

11 Filippidis FT, Tzoulaki I. Greece giving up on tobacco control. Addiction 2016; 111: 1306-1307.

12 Manoli E, Kouras A, Karagkiozidou O, et al. Polycyclic aromatic hydrocarbons (PAHs) at traffic and urban background sites of northern Greece: source apportionment of ambient PAH levels and PAH-induced lung cancer risk. Environ Sci Pollut Res Int 2016; 23: 3556-3568.

13 Tsiligianni IG. COPD patients trapped in the financial crisis in rural Crete. Lancet Respir Med 2013; 1: e31-e32.

14 Bakogeorgos M, Mountzios G, Bournakis E, et al. Do elderly patients with non-small cell lung cancer get the best out of recent advances in first-line treatment? A comparative study in two tertiary cancer centers in Greece. J Geriatr Oncol 2015; 6: 111-118.

15 Kalkhoran S, Glantz SA. Smoke-free policies: cleaning the air with money to spare. Lancet 2014; 383: 1526-1528.

\section{Acromegaly in sleep apnoea patients: a large observational study of 755 patients}

\section{To the Editor:}

Acromegaly is a rare, chronic and progressive disease characterised by excess secretion of growth hormone with raised insulin-like growth factor I (IGF-I) levels and usually caused by a pituitary adenoma $[1,2]$. Its prevalence is estimated at $40-480$ cases per million, depending on the study $[1,3]$. The diagnosis of acromegaly, frequently made late because of the insidious nature of the disease, is generally based on symptoms of excess growth hormone, such as acral enlargement, soft-tissue swelling, arthralgia, jaw prognathism, hyperhidrosis, osteoarthritis and frontal bossing, or symptoms of a pituitary adenoma, such as headaches, visual defects or pituitary insufficiency. Owing to prolonged untreated progression of the condition, patients often exhibit established systemic complications at diagnosis, such as diabetes mellitus (19$56 \%$ ) [2], hypertension (30-40\%) and respiratory/cardiac failure $(60 \%)$ [4, 5], which are the main determinants of prognosis and premature mortality [1]. The majority of new diagnoses of acromegaly are detected by primary care physicians, or specialists other than endocrinologists. The therapeutic options for acromegaly include endoscopic surgery, medical therapies (long-acting somatostatin analogues; dopamine agonists; growth hormone receptor antagonists) and pituitary radiotherapy [6, 7]. 45-80\% of acromegaly 
patients have sleep apnoea syndrome (SAS) [4,5], compared to $5 \%$ in the general population. Because of swelling of the uvula, macroglossia and maxillofacial modifications, obstructive sleep apnoea (OSA) is the prevailing form of SAS [8]. The combination of OSA and acromegaly increases the risk of systemic comorbidities and mortality [9]. The high prevalence of acromegaly-related comorbidities and its often late diagnosis [10], when valid treatment options exist, make screening for acromegaly in at-risk populations necessary. The OSA population might constitute a target group for the earlier detection of acromegaly, thereby preventing the late complications of the disease. Moreover, early treatment of acromegaly may favour the resolution of associated SAS. We prospectively assessed the prevalence of undiagnosed acromegaly in new patients suspected of suffering from OSA.

Between November 2013 and October 2014, we prospectively included adults referred for clinical suspicion of OSA to a tertiary teaching hospital or to one of 10 sleep clinics. All participants signed a written informed consent and the study was approved by the Grenoble Ethics Committee (CPP Sud-Est V, Grenoble, France; institutional review board number: 13-CHUG-37). During the baseline visit for suspected OSA, the Sleep registry of the French Federation of Pneumology (www.osfp.fr) was completed: sleep study results; Epworth Sleepiness Scale; fatigue and depression scales; medications; and pulmonary function test results. We also collected the clinical symptoms of acromegaly: acral enlargement; headache or visual field defects; facial changes; widely spaced teeth; diabetes; hyperhidrosis; arthralgia and/or arthritis; asthenia; menstrual disorders or galactorrhoea; carpal tunnel syndrome and acroparaesthesia; cardiovascular comorbidities (hypertension, diabetes); and prescribed lipid profile, fasting blood glucose and IGF-I tests. When serum IGF-I was elevated for age and sex, a new blood sample was requested and analysed for IGF-I level together with growth hormone monitoring during an oral glucose tolerance test (OGTT). If abnormal, patients were referred to an endocrinologist.

Assuming a prevalence of acromegaly of $0.04 \%$ in the general population [3] with a 10 -fold higher prevalence in the OSA population, we needed 880 patients (risk $\alpha 0.10$; margin of error \pm 0.35 ). We actually included 873 patients: 817 had laboratory IGF-I measurements and 755 underwent sleep studies to diagnose SAS and determine its severity. The study population had a median (interquartile range) age of 53 (44-61) years, were overweight or obese (median body mass index $29.8 \mathrm{~kg} \cdot \mathrm{m}^{-2}(26.1 \pm 4.5)$ ), predominantly male (63.9\%) and had a median (interquartile range) apnoea-hypopnoea index (AHI) of 25.3 (15-41) events $\cdot \mathrm{h}^{-1}$. OSA was confirmed in 567 patients (moderate-to-severe OSA, AHI $\geqslant 15$ events $\cdot \mathrm{h}^{-1}$ ).

Eight patients had IGF-I levels $>110 \%$ of the expected values for age and sex, including four with normal growth hormone profile during OGTT. Two patients were diagnosed with acromegaly after consultation with an endocrinologist and in both cases magnetic resonance imaging revealed a pituitary macroadenoma. Both also had severe OSA. The overall prevalence of acromegaly in patients with suspected SAS was $0.25 \%$ (90\% CI $0.05-0.93 \%)$. Among patients with confirmed OSA, the prevalence of acromegaly was $0.35 \%$ (90\% CI $0.08-1.19 \%)$.

More than $50 \%$ patients (both confirmed OSA and others) reported at least three symptoms suggesting growth hormone/IGF-I hypersecretion (table 1). Hypertension, diabetes and facial changes were significantly more frequent in those with $\mathrm{AHI} \geqslant 15$ events $\mathrm{h}^{-1}$. The prevalence of headaches and fatigue was very high in the whole study population but significantly higher in the non-OSA population, these symptoms being frequently reasons for referral to a sleep specialist.

Previous studies that screened for acromegaly in the general population [11], and in hypertensive [12], diabetic [3] or carpal tunnel syndrome patients [13] found similar results to ours. In patients with existing comorbidities, the only clinical indicators of acromegaly are obvious facial dysmorphism and enlargement of the extremities. This is reflected in the current guidelines for diagnosis that recommend IGF-I assays only in patients presenting typical acral and facial manifestations of acromegaly [7].

In view of the lack of compelling evidence supporting the value of systematic serum IGF-I measurement in the most commonly encountered comorbidities of acromegaly, the current consensus statement is relatively vague. It suggests "measurement of IGF-1 in patients without the typical manifestations of acromegaly, but who have several of these associated conditions: SAS, type 2 diabetes mellitus, debilitating arthritis, carpal tunnel syndrome, hyperhidrosis, and hypertension" [7]. The statement supports the use of a combination of signs, symptoms and comorbidities to screen for acromegaly but, to date, the exact cluster of comorbidities has not been validated.

Nevertheless, the at-risk OSA population seems one of the most promising for efficient screening of acromegaly. Our finding of an 8-30-fold increased prevalence of acromegaly in the OSA population compared to previously published data on other populations is explained by the fact that, over time, raised levels of IGF-I directly impact on craniofacial structures and swelling of soft tissues that are central to the pathophysiology of OSA. We recognise that the two patients diagnosed with acromegaly already exhibited 
TABLE 1 Comparison of symptoms and clinical signs of acromegaly in non-OSA and moderate-to-severe OSA groups (with insulin-like growth factor I measurement and sleep studies) ${ }^{\#}$

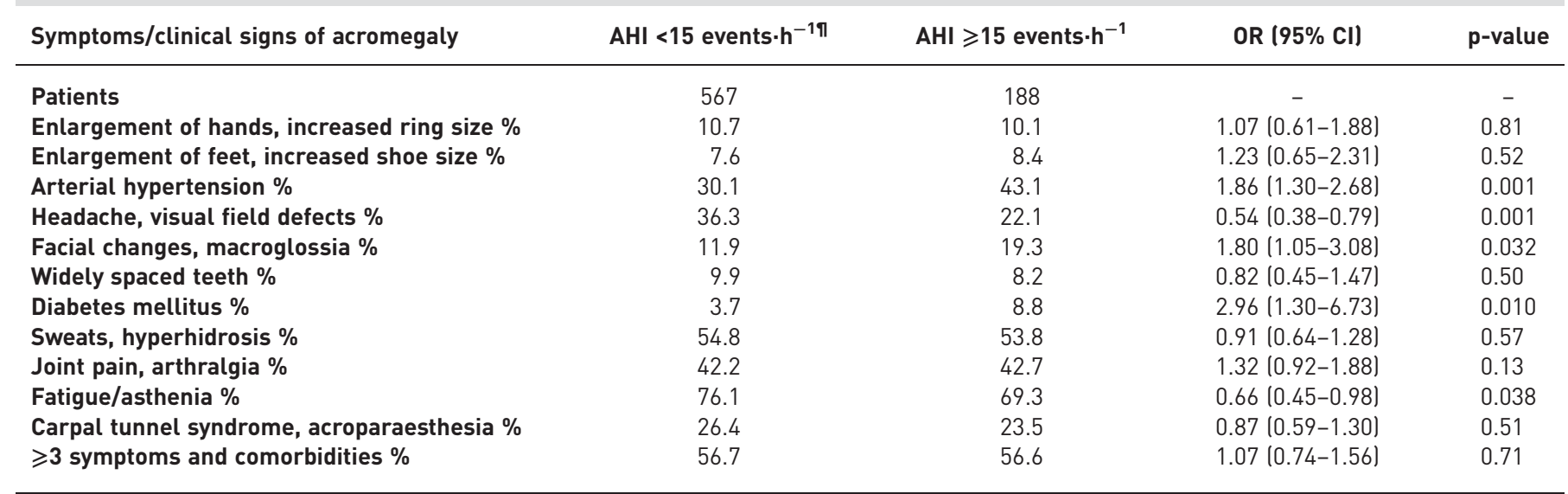

${ }^{\#}: \mathrm{n}=755 ;$ " : the non-OSA group consisted of the patients referred for suspicion of sleep apnoea syndrome, but with a diagnosis of no or only mild OSA (AHI < 15 events. $\mathrm{h}^{-1}$ ). AHI: apnoea-hypopnoea index; IGF-I: insulin-like growth factor I; OSA: obstructive sleep apnoea.

facial and acral dysmorphism. Such a small number of cases with typical acromegaly did not allow us to address the true challenge: detecting the very early phase of the disease. Not only would early diagnosis of acromegaly prevent irreversible comorbidities and facilitate surgical cures, but it would also permit the normalisation of OSA thereby avoiding long-term continuous positive airway pressure treatment. Trans-sphenoidal surgery and long-acting somatostatin analogues [8] are associated with a significant improvement in OSA severity, although up to $40 \%$ of those with cured or controlled acromegaly continue to exhibit significant OSA.

The level of awareness and training of sleep specialists and respiratory physicians needs to be improved to enable them to recognise the typical acromegaly phenotype in routine practice, so as to indicate an IGF-I measurement. In France, to measure IGF-I in 1000 patients would cost about USD 33000. If IGF-I screening permitted early diagnosis of acromegaly leading to a complete surgical cure, the cost-saving for society would be considerable (cost of surgery versus lifetime somatostatin analogue monotherapy: USD 15216 and USD 1938000, respectively) [14]. The justification for more general screening, especially in the absence of the typical phenotype, requires well-conducted, cost-effectiveness studies.

$\boldsymbol{0}$ @ERSpublications Among patients with confirmed obstructive sleep apnoea the prevalence of acromegaly was $0.35 \%$ http://ow.ly/eyrq302v4AI

Louis-Marie Galerneau $^{1,7}$, Jean-Louis Pépin ${ }^{1,7}$, Anne-Laure Borel ${ }^{1,2}$, Olivier Chabre ${ }^{2}$, Marc Sapene ${ }^{3}$, Bruno Stach ${ }^{4}$, Janie Girey-Rannaud ${ }^{5}$, Nathalie Arnol ${ }^{1}$, Renaud Tamisier ${ }^{1}$ and Philippe Caron ${ }^{6}$, on behalf of the scientific council and investigators of the French national sleep apnoea registry (OSFP)

${ }^{1}$ Institut national de la santé et de la recherche médicale (INSERM), U 1042, HP2 Laboratory (Hypoxia: Pathophysiology), Université Grenoble Alpes, Grenoble, France. ${ }^{2}$ Endocrinology Dept, Pôle Digidune, Grenoble Alpes University Hospital, Digidune, France. ${ }^{3}$ Centre de pneumologie Rivière, Bordeaux, France. ${ }^{4}$ Clinique Tessier, Valenciennes, France. ${ }^{5}$ Cabinet Pneumologie, Grenoble, France. ${ }^{6}$ Dept of Endocrinology, CHU Larrey, Pôle CardioVasculaire et Métabolisme, CHU Larrey, Toulouse, France. ${ }^{7}$ Both authors contributed equally.

Correspondence: Jean-Louis Pépin, Laboratoire EFCR, CHU de Grenoble, CP 1021738043 Grenoble Cedex 09, France. E-mail: jpepin@chu-grenoble.fr

Received: May 242016 | Accepted after revision: June 132016 | First published online: Sept 222016

Support statement: This study received unrestricted grants from Ipsen (France) and the endowment fund "Agir pour les maladies chroniques". Funding information for this article has been deposited with the Open Funder Registry.

Conflict of interest: Disclosures can be found alongside this article at erj.ersjournals.com

Acknowledgements: We thank Alison Foote (Grenoble Alpes University Hospital, Grenoble, France) for rewriting the manuscript.

Authors' contributions: J-L. Pépin had full access to all of the data in the study, takes responsibility for the content of the manuscript, including the data and analysis, and contributed to the study hypothesis, study design, data collection and 
analyses, writing the manuscript and sharing scientific discussions. L-M. Galerneau contributed to the study design, data collection and analyses, writing the manuscript and sharing scientific discussions. A-L. Borel contributed to the study design, analyses and revising the manuscript. O. Chabre contributed to the study hypothesis, study design, data collection and analyses, revising the manuscript and sharing scientific discussions. M. Sapene, B. Stach and J. Girey-Rannaud contributed to the study hypothesis, study design and data collection. R. Tamisier and P. Caron contributed to the study hypothesis, study design, data collection and analyses, writing the manuscript and sharing scientific discussions. N. Arnol performed the statistical analyses and contributed to revising the manuscript.

Prior abstract publication/presentation: French National Sleep Congress, Nantes, November 2015; American Thoracic Society Conference, San Francisco, May 2016.

\section{References}

Melmed S. Medical progress: acromegaly. N Engl J Med 2006; 355: 2558-2573.

Lugo G, Pena L, Cordido F. Clinical manifestations and diagnosis of acromegaly. Int J Endocrinol 2012; 2012: 540398.

3 Rosario PW. Frequency of acromegaly in adults with diabetes or glucose intolerance and estimated prevalence in the general population. Pituitary 2011; 14: 217-221.

4 Fatti LM, Scacchi M, Pincelli AI, et al. Prevalence and pathogenesis of sleep apnea and lung disease in acromegaly. Pituitary 2001; 4: 259-262.

5 Attal P, Chanson P. Endocrine aspects of obstructive sleep apnea. J Clin Endocrinol Metab 2010; 95: 483-495.

6 Abu Dabrh AM, Mohammed K, Asi N, et al. Surgical interventions and medical treatments in treatment-naïve patients with acromegaly: systematic review and meta-analysis. J Clin Endocrinol Metab 2014; 99: 4003-4014.

7 Katznelson L, Laws ER Jr, Melmed S, et al. Acromegaly: an endocrine society clinical practice guideline. J Clin Endocrinol Metab 2014; 99: 3933-3951.

8 Ip MS, Tan KC, Peh WC, et al. Effect of Sandostatin LAR on sleep apnoea in acromegaly: correlation with computerized tomographic cephalometry and hormonal activity. Clin Endocrinol (Oxf) 2001; 55: 477-483.

9 Colao A, Ferone D, Marzullo P, et al. Systemic complications of acromegaly: epidemiology, pathogenesis, and management. Endocr Rev 2004; 25: 102-152.

10 Reid TJ, Post KD, Bruce JN, et al. Features at diagnosis of 324 patients with acromegaly did not change from 1981 to 2006: acromegaly remains under-recognized and under-diagnosed. Clin Endocrinol (Oxf) 2010; 72: 203-208.

11 Rosario PW, Calsolari MR. Screening for acromegaly by application of a simple questionnaire evaluating the enlargement of extremities in adult patients seen at primary health care units. Pituitary 2012; 15: 179-183.

12 Rosario PW, Calsolari MR. Screening for acromegaly in adult patients not reporting enlargement of the extremities, but with arterial hypertension associated with another comorbidity of the disease. Arq Bras Endocrinol Metabol 2014; 58: 807-811.

13 Zoicas F, Kleindienst A, Mayr B, et al. Screening for acromegaly in patients with carpal tunnel syndrome: a prospective study (ACROCARP). Horm Metab Res 2016; 48: 452-456.

14 Kimmell KT, Weil RJ, Marko NF. Multi-modal management of acromegaly: a value perspective. Pituitary 2015; 18: 658-665.

\section{Introduction of the 13-valent pneumococcal conjugate vaccine in an isolated pneumococcal vaccine-naïve indigenous population}

To the Editor:

The introduction of pneumococcal conjugate vaccines (PCVs) has the greatest impact in populations that are most affected by pneumococcal carriage and disease, such as indigenous children [1]. Although $~ 10 \%$ of the South American population consists of indigenous people living in remote settings, to our knowledge PCVs have not been evaluated in native South American children. The Warao people are an Amerindian population residing in wooden houses along the Orinoco River delta in Venezuela. Almost one-third of Warao children die during childhood and respiratory tract infections are a major cause of death [2]. This study is the first to evaluate the impact of 13-valent (PCV13) vaccination on nasopharyngeal colonisation rates and antibody response in PCV-naïve indigenous South American children.

From May to November 2012, 504 Warao children aged 6 weeks to 59 months residing in nine communities were vaccinated. Before and at a median 6.7 weeks after primary PCV13 series we obtained nasopharyngeal swabs $(n=424)$ and serum samples $(n=421)$. The primary series consisted of three vaccine doses for children 